\title{
RADEMACHER FUNCTIONS IN WEIGHTED SYMMETRIC SPACES
}

\author{
SERGEY ASTASHKIN
}

\begin{abstract}
The closed span of Rademacher functions is investigated in the weighted spaces $X(w)$, where $X$ is a symmetric space on $[0,1]$ and $w$ is a positive measurable function on $[0,1]$. By using the notion and properties of the Rademacher multiplicator space of a symmetric space, we give a description of the weights $w$ for which the Rademacher orthogonal projection is bounded in $X(w)$.
\end{abstract}

\section{Introduction}

We recall that the Rademacher functions on $[0,1]$ are defined by $r_{k}(t)=\operatorname{sign}\left(\sin 2^{k} \pi t\right)$ for every $t \in[0,1]$ and each $k \in \mathbb{N}$. It is well known that $\left\{r_{k}\right\}$ is an incomplete orthogonal system of independent random variables. This system plays a prominent role in the modern theory of Banach spaces and operators (see, e.g., [11, [12], [17] and [19]).

A classical result of Rodin and Semenov [20] states that the sequence $\left\{r_{k}\right\}$ is equivalent in a symmetric space $X$ to the unit vector basis in $\ell_{2}$, i.e.,

$$
\left\|\sum_{k=1}^{\infty} a_{k} r_{k}\right\|_{X} \asymp\left(\sum_{k=1}^{\infty}\left|a_{k}\right|^{2}\right)^{1 / 2}, \quad\left(a_{k}\right) \in \ell_{2},
$$

if and only if $G \subset X$, where $G$ is the closure of $L_{\infty}[0,1]$ in the Zygmund space $\operatorname{Exp} L^{2}[0,1]$. When this condition is satisfied, the span $\left[r_{k}\right]$ of Rademacher functions is complemented in $X$ if and only if $X \subset G^{\prime}$, where the Köthe dual space $G^{\prime}$ to $G$ coincides (with equivalence of norms) with another wellknown Zygmund space $L \log ^{1 / 2} L[0,1]$. This was proved independently by Rodin and Semenov [21] and Lindenstrauss and Tzafriri [15, pp.138-138]. Moreover, the condition $G \subset X \subset G^{\prime}$ (equivalently, complementability of $\left[r_{k}\right]$ in $X$ ) is equivalent to the boundedness in $X$ of the orthogonal projection

$$
P f(t):=\sum_{k=1}^{\infty} c_{k}(f) r_{k}(t),
$$

where $c_{k}(f):=\int_{0}^{1} f(u) r_{k}(u) d u, k=1,2, \ldots$ The main purpose of this paper is to investigate the behaviour of Rademacher functions and of the respective projection $P$ in the weighted spaces $X(w)$ consisting of all measurable functions $f$ such that $f w \in X$ with the norm $\|f\|_{X(w)}:=\|f w\|_{X}$. Here, $X$ is a symmetric space on $[0,1]$ and $w$ is a positive measurable function on $[0,1]$. We make use of the notion of the Rademacher multiplicator space $\mathcal{M}(X)$ of a symmetric space $X$, which originally arised from the study of vector measures and scalar functions integrable with respect to them (see [8] and [10]). For the first time a connection between the space $\mathcal{M}(X)$ and the behavior 
of Rademacher functions in the weighted spaces $X(w)$ was observed in [6] when proving a weighted version of inequality (11) (under more restrictive conditions in the case of $L_{p}$-spaces it was proved in [23]).

To ensure that the operator $P$ is well defined, we have to guarantee that the Rademacher functions belong both to $X(w)$ and to its Köthe dual space $(X(w))^{\prime}=X^{\prime}(1 / w)$. For this reason, in what follows we assume that

$$
L_{\infty} \subset X(w) \subset L_{1} .
$$

This assumption allows us to find necessary and sufficient conditions on the weight $w$ under which the orthogonal projection $P$ is bounded in the weighted space $X(w)$. Moreover, extending above mentioned result of Rodin and Semenov from [20] to the weighted symmetric spaces, we show that, in contrast to the symmetric spaces, the embedding $X(w) \supset G$ is a stronger condition, in general, than equivalence of the sequence of Rademacher functions in $X(w)$ to the unit vector basis in $\ell_{2}$. In the final part of the paper, answering a question from [10], we present a concrete example of a function $f \in \mathcal{M}\left(L_{1}\right)$, which does not belong to the symmetric kernel of the latter space.

\section{Preliminaries}

Let $E$ be a Banach function lattice on $[0,1]$, i.e., if $x$ and $y$ are measurable a.e. finite functions on $[0,1]$ such that $x \in E$ and $|y| \leq|x|$, then $y \in E$ and $\|y\|_{E} \leq\|x\|_{E}$. The Köthe dual of $E$ is the Banach function lattice $E^{\prime}$ of all functions $y$ such that $\int_{0}^{1}|x(t) y(t)| d t<\infty$, for every $x \in E$, with the norm

$$
\|y\|_{E^{\prime}}:=\sup \left\{\int_{0}^{1} x(t) y(t) d t: x \in E,\|x\|_{E} \leq 1\right\} .
$$

$E^{\prime}$ is a subspace of the topological dual $E^{*}$. If $E$ is separable we have $E^{\prime}=E^{*}$. A Banach function lattice $E$ has the Fatou property, if from $0 \leq x_{n} \nearrow x$ a.e. on $[0,1]$ and $\sup _{n \in \mathbf{N}}\left\|x_{n}\right\|_{E}<\infty$ it follows that $x \in E$ and $\left\|x_{n}\right\|_{E} \nearrow\|x\|_{E}$.

Suppose a Banach function lattice $E \supset L_{\infty}$. By $E_{\circ}$ we will denote the closure of $L_{\infty}$ in $E$. Clearly, $E_{\circ}$ contains the absolutely continuous part of $E$, that is, the set of all functions $x \in E$ such that $\lim _{m(A) \rightarrow 0}\left\|x \cdot \chi_{A}\right\|_{E}=0$. Here and next, $m$ is the Lebesgue measure on $[0,1]$ and $\chi_{A}$ is the characteristic function of a set $A \subset[0,1]$.

Throughout the paper a symmetric (or rearrangement inveriant) space $X$ is a Banach space of classes of measurable functions on $[0,1]$ such that from the conditions $y^{*} \leq x^{*}$ and $x \in X$ it follows that $y \in X$ and $\|y\|_{X} \leq\|x\|_{X}$. Here, $x^{*}$ is the decreasing rearrangement of $x$, that is, the right continuous inverse of its distribution function: $n_{x}(\tau)=m\{t \in[0,1]:|x(t)|>\tau\}$ Functions $x$ and $y$ are said to be equimeasurable if $n_{x}(\tau)=n_{y}(\tau)$, for all $\tau>0$. The Köthe dual $X^{\prime}$ is a symmetric space whenever $X$ is symmetric. In what follows we assume that $X$ is isometric to a subspace of its second Köthe dual $X^{\prime \prime}:=\left(X^{\prime}\right)^{\prime}$. In particular, this holds if $X$ is separable or it has the Fatou property. For every symmetric space $X$ the following continuous embeddings hold: $L_{\infty} \subset X \subset L_{1}$. If $X$ is a symmetric space, $X \neq L_{\infty}$, then $X_{\circ}$ is a separable symmetric space.

Important examples of symmetric spaces are Marcinkiewicz, Lorentz and Orlicz spaces. Let $\varphi:[0,1] \rightarrow[0,+\infty)$ be a quasi-concave function, that is, $\varphi$ increases, $\varphi(t) / t$ decreases and $\varphi(0)=0$. The Marcinkiewicz space $M(\varphi)$ is the space of all measurable functions $x$ on $[0,1]$ for which the norm

$$
\|x\|_{M(\varphi)}=\sup _{0<t \leq 1} \frac{\varphi(t)}{t} \int_{0}^{t} x^{*}(s) d s<\infty .
$$


If $\varphi:[0,1] \rightarrow[0,+\infty)$ is an increasing concave function, $\varphi(0)=0$, then the Lorentz space $\Lambda(\varphi)$ consists of all measurable functions $x$ on $[0,1]$ such that

$$
\|x\|_{\Lambda(\varphi)}=\int_{0}^{1} x^{*}(s) d \varphi(s)<\infty .
$$

For arbitrary increasing convex function $\varphi$ we have $\Lambda(\varphi)^{\prime}=M(\tilde{\varphi})$ and $M(\varphi)^{\prime}=\Lambda(\tilde{\varphi})$, where $\tilde{\varphi}(t):=t / \varphi(t)$ [14, Theorems II.5.2 and II.5.4].

Let $M$ be an Orlicz function, that is, an increasing convex function on $[0, \infty)$ with $M(0)=0$. The norm of the Orlicz space $L_{M}$ is defined as follows

$$
\|x\|_{L_{M}}=\inf \left\{\lambda>0: \int_{0}^{1} M\left(\frac{|x(s)|}{\lambda}\right) d s \leq 1\right\} .
$$

In particular, if $M(u)=u^{p}, 1 \leq p<\infty$, we have $L_{M}=L_{p}$ isometrically. Next, by $\|f\|_{p}$ we denote the norm $\|f\|_{L_{p}}$.

The fundamental function of a symmetric space $X$ is the function $\phi_{X}(t):=\left\|\chi_{[0, t]}\right\|_{X}$. In particular, we have $\phi_{M(\varphi)}(t)=\phi_{\Lambda(\varphi)}(t)=\varphi(t)$, and $\phi_{L_{M}}(t)=1 / M^{-1}(1 / t)$, respectively. The Marcinkiewicz $M(\varphi)$ and Lorentz $\Lambda(\varphi)$ spaces are, respectively, the largest and the smallest symmetric spaces with the fundamental function $\varphi$, that is, if the fundamental function of a symmetric space $\mathrm{X}$ is equal to $\varphi$, then $\Lambda(\varphi) \subset X \subset M(\varphi)$.

If $\psi$ is a positive function defined on $[0,1]$, then its lower and upper dilation indices are

$$
\gamma_{\psi}:=\lim _{t \rightarrow 0^{+}} \frac{\log \left(\sup _{0<s \leq 1} \frac{\psi(s t)}{\psi(s)}\right)}{\log t} \text { and } \delta_{\psi}:=\lim _{t \rightarrow+\infty} \frac{\log \left(\sup _{0<s \leq 1 / t} \frac{\psi(s t)}{\psi(s)}\right)}{\log t}
$$

respectively. Always we have $0 \leq \gamma_{\psi} \leq \delta_{\psi} \leq 1$.

In the case when $\delta_{\varphi}<1$ the norm in the Marcinkiewicz space $M(\varphi)$ satisfies the equivalence

$$
\|x\|_{M(\varphi)} \asymp \sup _{0<t \leq 1} \varphi(t) x^{*}(t)
$$

[14. Theorem II.5.3]. Here, and throughout the paper, $A \asymp B$ means that there exist constants $C>0$ and $c>0$ such that $c \cdot A \leq B \leq C \cdot A$.

The Orlicz spaces $L_{N_{p}}, p>0$, where $N_{p}$ is an Orlicz function equivalent to the function $\exp \left(t^{p}\right)-1$, will be of major importance in our study. Usually these are referred as the Zygmund spaces and denoted by $\operatorname{Exp} L^{p}$. The fundamental function of $\operatorname{Exp} L^{p}$ is equivalent to the function $\varphi_{p}(t)=$ $\log ^{-1 / p}(e / t)$. Since $N_{p}(u)$ increases at infinity very rapidly, $\operatorname{Exp} L^{p}$ coincides with the Marcinkiewicz space $M\left(\varphi_{p}\right)$ [16]. This, together with the equality $\delta_{\varphi_{p}}=0<1$, gives

$$
\|x\|_{\operatorname{Exp} L^{p}} \asymp \sup _{0<t \leq 1} x^{*}(t) \log ^{-1 / p}(e / t) .
$$

In particular, for every $x \in \operatorname{Exp} L^{p}$ and $0<t \leq 1$ we have

$$
x^{*}(t) \leq C\|x\|_{\operatorname{Exp} L^{p}} \log ^{1 / p}(e / t) .
$$

Hence, for a symmetric space $X$, the embedding $\operatorname{Exp} L^{p} \subset X$ is equivalent to the condition $\log ^{1 / p}(e / t) \in$ $X$.

Recall that the Rademacher functions are $r_{k}(t):=\operatorname{sign} \sin \left(2^{k} \pi t\right), t \in[0,1], k \geq 1$. The famous Khintchine inequality [13] states that, for every $1 \leq p<\infty$, the sequence $\left\{r_{k}\right\}$ is equivalent in $L_{p}$ 
to the unit vector basis in $\ell_{2}$. As was mentioned in Introduction, Rodin and Semenov extended this result to the class of symmetric spaces showing that inequality (10) holds in a symmetric space $X$ if and only if $G \subset X$, where $G=\left(\operatorname{Exp} L^{2}\right)_{\circ}$. [20]. Next, we will repeatedly use the Khintchine $L_{1}$-inequality with optimal constants:

$$
\frac{1}{\sqrt{2}}\left\|\left(a_{k}\right)\right\|_{\ell_{2}} \leq\left\|\sum_{k=1}^{\infty} a_{k} r_{k}\right\|_{1} \leq\left\|\left(a_{k}\right)\right\|_{\ell_{2}}
$$

(see [22]), where $\left\|\left(a_{k}\right)\right\|_{\ell_{2}}:=\left(\sum_{k=1}^{\infty}\left|a_{k}\right|^{2}\right)^{1 / 2}$.

The Rademacher multiplicator space of a symmetric space $X$ is the space $\mathcal{M}(X)$ of all measurable functions $f:[0,1] \rightarrow \mathbb{R}$ such that $f \cdot \sum_{k=1}^{\infty} a_{k} r_{k} \in X$, for every Rademacher sum $\sum_{k=1}^{\infty} a_{k} r_{k} \in X$. It is a Banach function lattice on $[0,1]$ when endowed with the norm

$$
\|f\|_{\mathcal{M}(X)}=\sup \left\{\left\|f \cdot \sum_{k=1}^{\infty} a_{k} r_{k}\right\|_{X}:\left\|\sum_{k=1}^{\infty} a_{k} r_{k}\right\|_{X} \leq 1\right\} .
$$

$\mathcal{M}(X)$ can be viewed as the space of operators given by multiplication by a measurable function, which are bounded from the subspace $\left[r_{k}\right]$ in $X$ into the whole space $X$.

The Rademacher multiplicator space $\mathcal{M}(X)$ was firstly considered in [9], where it was shown that for a broad class of classical symmetric spaces $X$ the space $\mathcal{M}(X)$ is not symmetric. This result was extended in [3] to include all symmetric spaces such that the lower dilation index $\gamma_{\varphi_{X}}$ of their fundamental function $\varphi_{X}$ is positive. This result motivated the study of the symmetric kernel $\operatorname{Sym}(X)$ of the space $\mathcal{M}(X)$. The space $\operatorname{Sym}(X)$ consists of all functions $f \in \mathcal{M}(X)$ such that an arbitrary function $g$, equimeasurable with $f$, belongs to $\mathcal{M}(X)$ as well. The norm in $\operatorname{Sym}(X)$ is defined as follows

$$
\|f\|_{\text {Sym }(X)}=\sup \|g\|_{\mathcal{M}(X)},
$$

where the supremum is taken over all $g$ equimeasurable with $f$. From the definition it follows that $\operatorname{Sym}(X)$ is the largest symmetric space embedded into $\mathcal{M}(X)$ (see also [3, Proposition 2.4]). Moreover, if $X$ is a symmetric space such that $X^{\prime \prime} \supset \operatorname{Exp} L^{2}$, then

$$
\|f\|_{\operatorname{Sym}(X)} \asymp\left\|f^{*}(t) \log ^{1 / 2}(e / t)\right\|_{X^{\prime \prime}}
$$

(see [5, Proposition 3.1 and Corollary 3.2]). The opposite situation is when the Rademacher multiplicator space $\mathcal{M}(X)$ is symmetric. The simplest case of this situation is when $\mathcal{M}(X)=L_{\infty}$. It was shown in [4] that $\mathcal{M}(X)=L_{\infty}$ if and only if $\log ^{1 / 2}(e / t) \notin X_{\circ}$. Regarding the case when $\mathcal{M}(X)$ is a symmetric space different from $L_{\infty}$ see the paper [5].

We will denote by $\Delta_{n}^{k}$ the dyadic intervals of $[0,1]$, that is, $\Delta_{n}^{k}=\left[(k-1) 2^{-n}, k 2^{-n}\right]$, where $n=0,1, \ldots, k=1, \ldots, 2^{n}$; we say that $\Delta_{n}^{k}$ has rank $n$. For any undefined notions we refer the reader to the monographs [7], [14], [15].

\section{Rademacher sums in weighted spaces}

First, we find necessary and sufficient conditions on the symmetric space $X$, under which there is a weight $w$ such that the sequence of Rademacher functions spans $\ell_{2}$ in $X(w)$. We prove the following refinement of the nontrivial part of above mentioned Rodin-Semenov theorem. 
Proposition 3.1. For every symmetric space $X$ the following conditions are equivalent:

(i) there exists a set $D \subset[0,1]$ of positive measure such that

$$
\left\|\sum_{k=1}^{\infty} a_{k} r_{k} \cdot \chi_{D}\right\|_{X} \leq M\left\|\left(a_{k}\right)\right\|_{\ell_{2}}
$$

for some $M>0$ and arbitrary $\left(a_{k}\right) \in \ell_{2}$;

(ii) $X \supset G$.

Proof. Since implication $(i i) \Rightarrow(i)$ is an immediate consequence of the fact that the sequence $\left\{r_{k}\right\}$ spans $\ell_{2}$ in the space $G$ (see [18] or [24, Theorem V.8.16]), we need to prove only that $(i)$ implies (ii).

Assume that (6) holds. By Lebesgue's density theorem, for sufficiently large $m \in \mathbb{N}$, we can find a dyadic interval $\Delta:=\Delta_{m}^{k_{0}}=\left[\left(k_{0}-1\right) 2^{-m}, k_{0} 2^{-m}\right]$ such that

$$
2^{-m}=m(\Delta) \geq m(\Delta \cap D)>2^{-m-1} .
$$

Let us consider the set $E=\bigcup_{k=1}^{2^{m}} E_{m}^{k}$, where $E_{m}^{k}$ is obtained by translating the set $\Delta \cap D$ to the interval $\Delta_{m}^{k}, k=1,2, \ldots, 2^{m}$, (in particular, $E_{m}^{k_{0}}=\Delta \cap D$ ). Denote $f_{i}=r_{i} \cdot \chi_{E}, i \in \mathbb{N}$. It follows easily that $\left|f_{i}(t)\right| \leq 1, t \in[0,1],\left\|f_{i}\right\|_{2} \geq 1 / \sqrt{2}$, and $f_{i} \rightarrow 0$ weakly in $L_{2}[0,1]$ when $i \rightarrow \infty$. Therefore, by [1, Theorem 5], the sequence $\left\{f_{i}\right\}_{i=1}^{\infty}$ contains a subsequence $\left\{f_{i_{j}}\right\}$, which is equivalent in distribution to the Rademacher system. The last means that there exists a constant $C>0$ such that

$$
\begin{aligned}
C^{-1} m\left\{t \in[0,1]:\left|\sum_{j=1}^{l} a_{j} r_{j}(t)\right|>C z\right\} & \leq m\left\{t \in[0,1]:\left|\sum_{j=1}^{l} a_{j} f_{i_{j}}(t)\right|>z\right\} \\
& \leq C m\left\{t \in[0,1]:\left|\sum_{j=1}^{l} a_{j} r_{j}(t)\right|>C^{-1} z\right\}
\end{aligned}
$$

for all $l \in \mathbb{N}, a_{j} \in \mathbb{R}$, and $z>0$. Hence, by the definition of $r_{j}$ and $f_{j}$, for every $n \in \mathbb{N}$ we have

$$
\begin{aligned}
C^{-1} m\{t \in[0,1]: \mid & \left.\sum_{j=m+1}^{m+n} r_{j}(t) \chi_{\left[0,2^{-m}\right]}(t) \mid>C z\right\} \\
& \leq m\left\{t \in[0,1]:\left|\sum_{j=m+1}^{m+n} f_{i_{j}}(t) \chi_{\Delta}(t)\right|>z\right\} \\
& \leq C m\left\{t \in[0,1]:\left|\sum_{j=m+1}^{m+n} r_{j}(t) \chi_{\left[0,2^{-m}\right]}(t)\right|>C^{-1} z\right\},
\end{aligned}
$$

whence

$$
\left\|\sum_{j=m+1}^{m+n} r_{i_{j}} \chi_{\Delta \cap D}\right\|_{X} \geq \alpha\left\|\sum_{j=m+1}^{m+n} r_{j} \chi_{\left[0,2^{-m}\right]}\right\|_{X},
$$

where $\alpha>0$ depends only on the constant $C$ and on the space $X$.

Now, assume that ( $i i)$ fails, i.e., $X \not \supset G$. Then, by [4, inequality (2) in the proof of Theorem 1], there exists a constant $\beta>0$, depending only on $X$, such that for every $m \geq 0$ there exists $n_{0} \geq 1$ 
such that, if $n \geq n_{0}$ and $\Delta$ is an arbitrary dyadic interval of rank $m$, we have

$$
\left\|\chi_{\Delta} \sum_{i=m+1}^{m+n} r_{i}\right\|_{X} \geq \beta\left\|\sum_{i=1}^{n} r_{i}\right\|_{X} .
$$

From this inequality with $\Delta=\left[0,2^{-m}\right]$ and inequality (7) it follows that, for $n$ large enough,

$$
\left\|\sum_{j=m+1}^{m+n} r_{i_{j}} \chi_{D}\right\|_{X} \geq\left\|\sum_{j=m+1}^{m+n} r_{i_{j}} \chi_{\Delta \cap D}\right\|_{X} \geq \alpha \beta\left\|\sum_{j=1}^{n} r_{j}\right\|_{X} .
$$

Combining the latter inequality together with (6) we deduce

$$
\frac{1}{\sqrt{n}}\left\|\sum_{j=1}^{n} r_{j}\right\|_{X} \leq \frac{M}{\alpha \beta}
$$

for all $n \in \mathbb{N}$ large enough. At the same time, as it follows from the proof of Rodin-Semenov theorem [20], the last condition is equivalent to the embedding $X \supset G$. This contradiction concludes the proof.

Corollary 3.1. Suppose $X$ is a symmetric space. Then, $X \supset G$ if and only if there exists a weight $w$ such that the sequence $\left\{r_{k}\right\}$ spans $\ell_{2}$ in $X(w)$.

Proof. If $\left\{r_{k}\right\}$ spans $\ell_{2}$ in $X(w)$ for some weight $w$, we have

$$
\left\|\sum_{k=1}^{\infty} a_{k} r_{k} \cdot w\right\|_{X} \leq C\left\|\left(a_{k}\right)\right\|_{\ell_{2}} .
$$

Since $w(t)>0$ a.e. on $[0,1]$, there is a set $D \subset[0,1]$ of positive measure such that inequality (6) holds for some $M>0$ and arbitrary $\left(a_{k}\right) \in \ell_{2}$. Applying Proposition 3.1, we obtain that $X \supset G$. The converse is obvious, and so the proof is completed.

Corollary 3.1 shows the necessity of the condition $X \supset G$ in the following main result of this part of the paper.

Theorem 3.1. Let $X$ be a symmetric space such that $X \supset G$ and let a positive measurable function $w$ on $[0,1]$ satisfy condition (3). Then we have

(i) The sequence $\left\{r_{k}\right\}$ spans $\ell_{2}$ in $X(w)$ if and only if $w \in \mathcal{M}(X)$, where $\mathcal{M}(X)$ is the Rademacher multiplicator space of $X$;

(ii) $X(w) \supset G$ if and only if $w \in \operatorname{Sym}(X)$, where $\operatorname{Sym}(X)$ is the symmetric kernel of $\mathcal{M}(X)$.

The part (i) of this theorem was actually obtained in [6, p. 240]. However, for the reader's convenience we provide here its proof. But we begin with the following technical result, which will be needed us to prove the part (ii).

Lemma 3.1. Let $Y$ be a symmetric space and $w$ be a positive measurable function on $[0,1]$. Suppose the weighted function lattice $Y\left(w^{*}\right)$ contains an unbounded decreasing positive function a on $(0,1]$. Then $(Y(w))_{\circ}=Y_{\circ}(w)$. 
Proof. Since $(w a)^{*}(t) \leq w^{*}(t / 2) a(t / 2), 0<t \leq 1$, [14, $\S$ II.2] and, by assumption, $w^{*} a \in Y$, we have $w a \in Y$. Equivalently, $a \in Y(w)$.

Let $y \in(Y(w))_{\circ}$. By definition, there is a sequence $\left\{y_{k}\right\} \subset L_{\infty}$ such that

$$
\lim _{k \rightarrow \infty}\left\|y_{k} w-y w\right\|_{Y}=0 .
$$

Show that $y_{k} w \in Y_{\circ}$ for every $k \in \mathbb{N}$.

Since $a$ decreases, for arbitrary $A \subset[0,1]$ and every (fixed) $k \in \mathbb{N}$ we have

$$
\left\|y_{k} w \chi_{A}\right\|_{Y} \leq\left\|y_{k}\right\|_{\infty}\left\|w^{*} \chi_{(0, m(A)]}\right\|_{Y} \leq \frac{\left\|y_{k}\right\|_{\infty}}{a(m(A))}\left\|w^{*} a\right\|_{Y}
$$

Noting that the right hand side of this inequality tends to 0 as $m(A) \rightarrow \infty$, we get

$$
\lim _{m(A) \rightarrow 0}\left\|y_{k} w \chi_{A}\right\|_{Y}=0
$$

whence $y_{k} w \in Y_{\circ}, k \in \mathbb{N}$. Combining this with (8), we infer that $y w \in Y_{\circ}$ or, equivalently, $y \in Y_{\circ}(w)$.

To prove the opposite embedding, assume that $y \in Y_{\circ}(w)$. Then

$$
\lim _{k \rightarrow \infty}\left\|y_{k}-y w\right\|_{Y}=0
$$

for some sequence $\left\{y_{k}\right\} \subset L_{\infty}$. From hypothesis of lemma it follows that $Y \neq L_{\infty}$. Therefore, for arbitrary $A \subset[0,1]$ and each $k \in \mathbb{N}$

$$
\left\|y_{k} / w \cdot \chi_{A}\right\|_{Y(w)}=\left\|y_{k} \chi_{A}\right\|_{Y} \rightarrow 0 \text { as } m(A) \rightarrow 0 .
$$

Hence, $y_{k} / w \in(Y(w))_{\circ}, k \in \mathbb{N}$. Since $\left\|y_{k} / w-y\right\|_{Y(w)}=\left\|y_{k}-y w\right\|_{Y}$, from (9) it follows that $y \in(Y(w))_{\circ}$.

Proof of Theorem 3.1. (i) Since $X \supset G$, equivalence (11) holds. At first, assume that $w \in \mathcal{M}(X)$. Then, by definition of the norm in $\mathcal{M}(X)$, we have

$$
\|w\|_{\mathcal{M}(X)} \asymp \sup \left\{\left\|w \cdot \sum_{k=1}^{\infty} a_{k} r_{k}\right\|_{X}:\left\|\left(a_{k}\right)\right\|_{\ell_{2}} \leq 1\right\} .
$$

Therefore,

$$
\left\|\sum_{k=1}^{\infty} a_{k} r_{k}\right\|_{X(w)}=\left\|w \cdot \sum_{k=1}^{\infty} a_{k} r_{k}\right\|_{X} \leq\|w\|_{\mathcal{M}(X)}\left\|\left(a_{k}\right)\right\|_{\ell_{2}}
$$

for every $\left(a_{k}\right) \in \ell_{2}$. On the other hand, from embeddings (3) and inequality (5) it follows that

$$
\left\|\sum_{k=1}^{\infty} a_{k} r_{k}\right\|_{X(w)} \geq c\left\|\sum_{k=1}^{\infty} a_{k} r_{k}\right\|_{1} \geq \frac{c}{\sqrt{2}}\left\|\left(a_{k}\right)\right\|_{\ell_{2}} .
$$

As a result we deduce that $\left\{r_{k}\right\}$ spans $\ell_{2}$ in $X(w)$.

Conversely, if

$$
\left\|\sum_{k=1}^{\infty} a_{k} r_{k}\right\|_{X(w)} \asymp\left\|\left(a_{k}\right)\right\|_{\ell_{2}},
$$

from (10) we obtain that $\|w\|_{\mathcal{M}(X)}<\infty$, i.e., $w \in \mathcal{M}(X)$. 
(ii) Assume that $w \in \operatorname{Sym}(X)$. Then, taking into account the properties of the symmetric kernel $\operatorname{Sym}(X)$ (see Preliminaries or [5, Corollary 3.2]) we have $w^{*}(t) \log ^{1 / 2}(e / t) \in X^{\prime \prime}$. Let us prove that

$$
\operatorname{Exp} L_{2} \subset X^{\prime \prime}(w)
$$

Given $x \in \operatorname{Exp} L_{2}$, by [7, Theorem 2.7.5], there exists a measure-preserving transformation $\sigma$ of $(0,1]$ such that $|x(t)|=x^{*}(\sigma(t))$. Applying inequality (44) and a well-known property of the rearrangement of a measurable function (see e.g. [14, § II.2]), we have

$$
(w x)^{*}(t)=\left(w x^{*}(\sigma)\right)^{*}(t) \leq C\left(w \log ^{1 / 2}(e / \sigma(\cdot))\right)^{*}(t) \leq C w^{*}(t / 2) \log ^{1 / 2}(2 e / t), \quad 0<t \leq 1 .
$$

Therefore, $w x \in X^{\prime \prime}$ or, equivalently, $x \in X^{\prime \prime}(w)$, and (111) is proved. Hence, $G=\left(\operatorname{Exp} L_{2}\right) \circ \subset$ $\left(X^{\prime \prime}(w)\right)_{\circ}$. Since $\log ^{1 / 2}(e / t) \in X^{\prime \prime}\left(w^{*}\right)$, we can apply Lemma 3.1] and so, by [2, Lemma 3.3],

$$
G \subset\left(X^{\prime \prime}\right)_{\circ}(w)=X_{\circ}(w) \subset X(w) .
$$

Now, let $X(w) \supset G$. We show that $X\left(w^{*}\right) \supset G$. In fact, let $\tau$ be a measure-preserving transformation of $(0,1]$ such that $w(t)=w^{*}(\tau(t))$ [7, Theorem 2.7.5]. Suppose $x \in G$. Since $x(\tau)$ and $x$ are equimesurable functions, we have $x(\tau) \in G$ and $\|x(\tau)\|_{G}=\|x\|_{G}$. Therefore,

$$
\left\|x(\tau) w^{*}(\tau)\right\|_{X}=\|x(\tau) w\|_{X} \leq C\|x\|_{G}
$$

Then, $\left\|x(\tau) w^{*}(\tau)\right\|_{X}=\left\|x w^{*}\right\|_{X}$, because $X$ is a symmetric space, and from the preceding inequality we infer that $\left\|x w^{*}\right\|_{X} \leq C\|x\|_{G}$. Thus, $x \in X\left(w^{*}\right)$, and the embedding $X\left(w^{*}\right) \supset G$ is proved. Passing to the second Köthe dual spaces, we obtain: $X^{\prime \prime}\left(w^{*}\right) \supset G^{\prime \prime}=\operatorname{Exp} L^{2}$. Hence, $\log ^{1 / 2}(e / t) \in X^{\prime \prime}\left(w^{*}\right)$ or, equivalently, $w \in \operatorname{Sym}(X)$ (as above, see Preliminaries or [5, Corollary 3.2]), and the proof is complete.

By Rodin-Semenov theorem [20, the sequence $\left\{r_{k}\right\}$ is equivalent in a symmetric space $X$ to the unit vector basis in $\ell_{2}$ if and only if $X \supset G$. In contrast to that from Theorem 3.1 we immediately deduce the following result.

Corollary 3.2. Suppose $X$ is a symmetric space such that $\operatorname{Sym}(X) \neq \mathcal{M}(X)$. Then, for every $w \in \mathcal{M}(X) \backslash \operatorname{Sym}(X)$ the Rademacher functions span $\ell_{2}$ in $X(w)$ but $X(w) \not \supset G$.

By [3. Theorem 2.1], $\operatorname{Sym}(X) \neq \mathcal{M}(X)$ ( and therefore there is $w \in \mathcal{M}(X) \backslash \operatorname{Sym}(X)$ ) whenever the lower dilation index of the fundamental function $\phi_{X}$ is positive. In particular, it is fulfilled for $L_{p}$-spaces, $1 \leq p<\infty$. The condition $\gamma_{\phi_{X}}>0$ means that the space $X$ is situated "far" from the minimal symmetric space $L_{\infty}$. Now, consider the opposite case when a symmetric space is "close" to $L_{\infty}$. Then the Rademacher multiplicator space $\mathcal{M}(X)$ may be symmetric (equivalently, it coincides with its symmetric kernel). Since the space $\operatorname{Sym}(X)$ has an explicit description (see Preliminaries), in this case we are able to state a sharper result. For simplicity, let us consider only Lorentz and Marcinkiewicz spaces (for more general results of such a sort see [5]).

Recall [5] that a function $\varphi(t)$ defined on $[0,1]$ satisfies the $\Delta^{2}$-condition (briefly, $\varphi \in \Delta^{2}$ ) if it is nonnegative, increasing, concave, and there exists $C>0$ such that $\varphi(t) \leq C \cdot \varphi\left(t^{2}\right)$ for all $0<t \leq 1$. By [5. Corollary 3.5], if $\varphi \in \Delta^{2}$, then $\mathcal{M}(\Lambda(\varphi))=\operatorname{Sym}(\Lambda(\varphi))$ and $\mathcal{M}(M(\varphi))=$ $\operatorname{Sym}(M(\varphi))$. Moreover, it is known [3, Example 2.15 and Theorem 4.1] that $\operatorname{Sym}(\Lambda(\varphi))=\Lambda(\psi)$ $(\operatorname{resp} . \operatorname{Sym}(M(\varphi))=M(\psi))$, where $\psi^{\prime}(t)=\varphi^{\prime}(t) \log ^{1 / 2}(e / t)$, whenever $\log ^{1 / 2}(e / t) \in \Lambda(\varphi)$ (resp. $\left.\log ^{1 / 2}(e / t) \in M(\varphi)\right)$. Therefore, we get 
Corollary 3.3. Let $\varphi \in \Delta^{2}$ and $\log ^{1 / 2}(e / t) \in \Lambda(\varphi)$ (resp. $\log ^{1 / 2}(e / t) \in M(\varphi)$ ). If $w$ is a positive measurable function on $[0,1]$ satisfying condition (3), then the sequence $\left\{r_{k}\right\}$ is equivalent in the space $\Lambda(\varphi)(w)$ (resp. $M(\varphi)(w)$ ) to the unit vector basis in $\ell_{2}$ if and only if $w \in \Lambda(\psi)$ (resp. $w \in M(\psi)$ ), where $\psi^{\prime}(t)=\varphi^{\prime}(t) \log ^{1 / 2}(e / t)$.

In particular, if $0<p \leq 2$, the sequence $\left\{r_{k}\right\}$ is equivalent in the Zygmund space $\operatorname{Exp} L^{p}(w)$ to the unit vector basis in $\ell_{2}$ if and only if $w \in \operatorname{Exp} L^{q}$, where $q=2 p /(2-p)$ (here, we set $\operatorname{Exp} L^{\infty}=L_{\infty}$ ).

\section{Rademacher orthogonal projection in weighted spaces}

Proposition 4.1. Let $E$ be a Banach function lattice on $[0,1]$ that is isometrically embedded into $E^{\prime \prime}, L_{\infty} \subset E \subset L_{1}$. Then the projection $P$ defined by (2) is bounded in $E$ if and only if there are constants $C_{1}$ and $C_{2}$ such that for all $a=\left(a_{k}\right) \in \ell_{2}$

$$
\left\|\sum_{k=1}^{\infty} a_{k} r_{k}\right\|_{E} \leq C_{1}\|a\|_{\ell_{2}}
$$

and

$$
\left\|\sum_{k=1}^{\infty} a_{k} r_{k}\right\|_{E^{\prime}} \leq C_{2}\|a\|_{\ell_{2}}
$$

Proof. Firstly, assume that inequalities (12) and (13) hold. Then, denoting, as above, $c_{k}(f):=$ $\int_{0}^{1} f(u) r_{k}(u) d u, k=1,2, \ldots$, for every $n \in \mathbb{N}$, by (13), we have

$$
\sum_{k=1}^{n} c_{k}(f)^{2}=\int_{0}^{1} f(u) \sum_{k=1}^{n} c_{k}(f) r_{k}(u) d u \leq\|f\|_{E}\left\|\sum_{k=1}^{n} c_{k}(f) r_{k}\right\|_{E^{\prime}} \leq C_{2}\|f\|_{E}\left(\sum_{k=1}^{n} c_{k}(f)^{2}\right)^{1 / 2}
$$

whence

$$
\left(\sum_{k=1}^{\infty} c_{k}(f)^{2}\right)^{1 / 2} \leq C_{2}\|f\|_{E}, \quad f \in E
$$

Therefore, by (12), we obtain

$$
\|P f\|_{E} \leq C_{1}\left(\sum_{k=1}^{\infty} c_{k}(f)^{2}\right)^{1 / 2} \leq C_{1} C_{2}\|f\|_{E}
$$

for all $f \in E$.

Conversely, suppose that the projection $P$ is bounded in $E$. Let us consider the following sequence of finite dimensional operators

$$
P_{n} f(t):=\sum_{k=1}^{n} c_{k}(f) r_{k}(t), \quad n \in \mathbb{N} .
$$

Clearly, $P_{n}$ is bounded in $E$ for every $n \in \mathbb{N}$. Furthermore, by assumption, the series $\sum_{k=1}^{\infty} c_{k}(f) r_{k}$ converges in $E$ for each $f \in E$. Therefore, by the Uniform Boundedness Principle,

$$
\left\|P_{n}\right\|_{E \rightarrow E} \leq B, \quad n \in \mathbb{N} .
$$


Moreover, since $L_{\infty} \subset E \subset L_{1}$, then $L_{\infty} \subset E^{\prime} \subset L_{1}$ as well, and hence, by the $L_{1}$-Khintchine inequality (5),

$$
\left\|\sum_{k=1}^{\infty} a_{k} r_{k}\right\|_{E} \geq c\|a\|_{\ell_{2}} \text { and }\left\|\sum_{k=1}^{\infty} a_{k} r_{k}\right\|_{E^{\prime}} \geq c\|a\|_{\ell_{2}} .
$$

Therefore, for all $f \in E, n \in \mathbb{N}$ and $a_{k} \in \mathbb{R}, k=1,2, \ldots, n$, we have

$$
\begin{aligned}
\int_{0}^{1} f(t) \cdot \sum_{k=1}^{n} a_{k} r_{k}(t) d t & =\sum_{k=1}^{n} a_{k} c_{k}(f) \leq\|a\|_{2}\left(\sum_{k=1}^{n} c_{k}(f)^{2}\right)^{1 / 2} \\
& \leq c^{-1}\|a\|_{\ell_{2}} \cdot\left\|P_{n} f\right\|_{E} \leq B c^{-1}\|a\|_{\ell_{2}} \cdot\|f\|_{E} .
\end{aligned}
$$

Taking the supremum over all $f \in E,\|f\|_{E} \leq 1$, we get

$$
\left\|\sum_{k=1}^{n} a_{k} r_{k}\right\|_{E^{\prime}} \leq B c^{-1}\|a\|_{\ell_{2}}, \quad n \in \mathbb{N} .
$$

Applying the latter inequality to Rademacher sums $\sum_{k=n}^{m} a_{k} r_{k}, 1 \leq n<m$, with $a=\left(a_{k}\right)_{k=1}^{\infty} \in \ell_{2}$, we deduce that the series $\sum_{k=1}^{\infty} a_{k} r_{k}$ converges in the space $E^{\prime}$ and

$$
\left\|\sum_{k=1}^{\infty} a_{k} r_{k}\right\|_{E^{\prime}} \leq B c^{-1}\|a\|_{\ell_{2}}
$$

Thus, (13) is proved. Let us prove similar inequality for $E$.

By Fubini theorem and (14), for arbitrary $f \in E, g \in E^{\prime}$ and every $n \in \mathbb{N}$ we have

$$
\int_{0}^{1} f(u) \cdot \sum_{k=1}^{n} c_{k}(g) r_{k}(u) d u=\int_{0}^{1} g(t) \cdot \sum_{k=1}^{n} c_{k}(f) r_{k}(t) d t \leq\left\|P_{n} f\right\|_{E}\|g\|_{E^{\prime}} \leq B\|f\|_{E}\|g\|_{E^{\prime}}
$$

whence

$$
\left\|\sum_{k=1}^{n} c_{k}(g) r_{k}\right\|_{E^{\prime}} \leq B\|g\|_{E^{\prime}}, \quad n \in \mathbb{N} .
$$

Applying this inequality instead of (14), as above, we get

$$
\left\|\sum_{k=1}^{n} a_{k} r_{k}\right\|_{E^{\prime \prime}} \leq B c^{-1}\|a\|_{\ell_{2}} .
$$

Since $L_{\infty} \subset E$ and $E$ is isometrically embedded into $E^{\prime \prime}$, from the last inequality it follows that

$$
\left\|\sum_{k=1}^{n} a_{k} r_{k}\right\|_{E} \leq B c^{-1}\|a\|_{\ell_{2}}
$$

for all $n \in \mathbb{N}$. Hence, if $a=\left(a_{k}\right)_{k=1}^{\infty} \in \ell_{2}$, the series $\sum_{k=1}^{\infty} a_{k} r_{k}$ converges in $E$ and

$$
\left\|\sum_{k=1}^{\infty} a_{k} r_{k}\right\|_{E} \leq B c^{-1}\|a\|_{\ell_{2}} .
$$

Thus, inequality (12) holds, and the proof is complete. 
From Proposition 4.1, Corollary 3.1 and Theorem 3.1 we obtain the following results.

Theorem 4.1. Let a symmetric space $X$ and a positive measurable function $w$ on $[0,1]$ satisfy condition (3). Then, the projection $P$ defined by (2) is bounded in $X(w)$ if and only if $G \subset X \subset G^{\prime}$, $w \in \mathcal{M}(X)$ and $1 / w \in \mathcal{M}\left(X^{\prime}\right)$. $X^{\prime}$.

In particular, $P$ is bounded in $X(w)$ whenever $w^{*}(t) \log ^{1 / 2}(e / t) \in X^{\prime \prime}$ and $(1 / w)^{*}(t) \log ^{1 / 2}(e / t) \in$

As above, the result can be somewhat refined for Lorentz and Marcinkiewicz spaces whose fundamental function satisfies the $\Delta^{2}$-condition.

Corollary 4.1. Let $\varphi \in \Delta^{2}$ and let $w$ be a positive measurable function on $[0,1]$ satisfying condition (3) for $X=\Lambda(\varphi)$ (resp. $X=M(\varphi)$ ). Then the projection $P$ defined by (2) is bounded in $\Lambda(\varphi)(w)$ (resp. $M(\varphi)(w)$ ) if and only if $G \subset \Lambda(\varphi) \subset G^{\prime}, w \in \Lambda(\psi)$ and $1 / w \in \mathcal{M}(M(\tilde{\varphi}))$ (resp. $G \subset M(\varphi) \subset$ $G^{\prime}, w \in M(\psi)$ and $\left.1 / w \in \mathcal{M}(\Lambda(\tilde{\varphi}))\right)$, where $\psi^{\prime}(t)=\varphi^{\prime}(t) \log ^{1 / 2}(e / t)$ and $\tilde{\varphi}(t)=t / \varphi(t)$.

Remark 4.1. It is easy to see that the orthogonal projection $P$ is bounded in the space $X(w)$ if and only if the projection

$$
P_{w} f(t):=\sum_{k=1}^{\infty} \int_{0}^{1} f(s) r_{k}(s) \frac{d s}{w(s)} \cdot r_{k}(t) w(t), \quad 0 \leq t \leq 1,
$$

(on the subspace $\left[r_{k} w\right]$ ) is bounded in $X$.

\section{$5 \quad$ Example of a function from $\mathcal{M}\left(L_{1}\right) \backslash \operatorname{Sym}\left(L_{1}\right)$}

Answering a question from [10], we present here a concrete example of a function $f \in \mathcal{M}\left(L_{1}\right)$, which does not belong to the symmetric kernel $\operatorname{Sym}\left(L_{1}\right)$, that is,

$$
\int_{0}^{1} f^{*}(t) \log ^{1 / 2}(e / t) d t=\infty .
$$

Since the latter space is symmetric, it is sufficient to find a function $f \in \mathcal{M}\left(L_{1}\right)$, for which there exists a function $g \notin \mathcal{M}\left(L_{1}\right)$ equimeasurable with $f$. We will look for $f$ and $g$ in the form

$$
f=\sum_{k=1}^{\infty} \alpha_{k} \chi_{B_{k}}, \quad g=\sum_{k=1}^{\infty} \alpha_{k} \chi_{D_{k}}
$$

where $\left\{B_{k}\right\}$ and $\left\{D_{k}\right\}$ are sequences of pairwise disjoint subsets of $[0,1], m\left(B_{k}\right)=m\left(D_{k}\right), \alpha_{k} \in \mathbb{R}$, $k=1,2, \ldots$ Next, we will make use of some ideas of the paper [9].

Let $n=2^{m}$ with $m \in \mathbb{N}$ and let $J$ be a subset of $\left\{1,2, \ldots, 2^{n}\right\}$ with cardinality $n$. We define the set $A=\bigcup_{j \in J} \Delta_{n}^{j}$ associated with $J$ (as above, $\Delta_{n}^{j}$ are the dyadic intervals of $[0,1]$ ). Clearly, $m(A)=n 2^{-n}$.

For arbitrary sequence $\left(b_{i}\right) \in \ell_{2}$ we have

$$
\left\|\chi_{A} \sum_{i=1}^{\infty} b_{i} r_{i}\right\|_{1} \leq\left\|\chi_{A} \sum_{i=1}^{n} b_{i} r_{i}\right\|_{1}+\left\|\chi_{A} \sum_{i=n+1}^{\infty} b_{i} r_{i}\right\|_{1} .
$$


Firstly, we estimate the tail term from the right hand side of this inequality. It is easy to see that the functions

$$
\chi_{A}(t) \cdot \sum_{i=n+1}^{\infty} b_{i} r_{i}(t) \text { and } \chi_{\left[0, n 2^{-n]}\right.}(t) \cdot \sum_{i=n+1}^{\infty} b_{i} r_{i}(t)
$$

are equimeasurable on $[0,1]$ and

$$
\chi_{\left[0, n 2^{-n]}\right.}(t) \sum_{i=n+1}^{\infty} b_{i} r_{i}(t)=\sum_{i=n+1}^{\infty} b_{i} r_{i+m-n}\left(n 2^{-n} t\right), \quad 0<t \leq 1 .
$$

Therefore,

$$
\left\|\chi_{A} \sum_{i=n+1}^{\infty} b_{i} r_{i}\right\|_{1}=\left\|\chi_{\left[0, n 2^{-n}\right]} \sum_{i=n+1}^{\infty} b_{i} r_{i}\right\|_{1}=n 2^{-n}\left\|\sum_{i=n+1}^{\infty} b_{i} r_{i+m-n}\right\|_{1} \leq n 2^{-n}\left(\sum_{i=n+1}^{\infty} b_{i}^{2}\right)^{1 / 2} .
$$

Now, choosing a set $A$ in a special way, estimate the first term from the right hand side of (16). Denote by $\varepsilon_{i j}^{n}$ the value of the function $r_{i}, i=1,2, \ldots, n$, on the interval $\Delta_{n}^{j}, 1 \leq j \leq 2^{n}$. Since $n=2^{m}$, we can find a set $J_{1}(n) \subset\left\{1,2, \ldots, 2^{n}\right\}$, card $J_{1}(n)=n$, such that the $n \times n$ matrix $n^{-1 / 2} \cdot\left(\varepsilon_{i j}^{n}\right)_{1 \leq i \leq n, j \in J_{1}(n)}$ is orthogonal. Then, if $c_{j}:=n^{-1 / 2} \sum_{i=1}^{n} \varepsilon_{i j}^{n} b_{i}, j \in J_{1}(n)$, we have $\left\|\left(c_{j}\right)_{j \in J_{1}(n)}\right\|_{\ell_{2}}=\left\|\left(b_{i}\right)_{i=1}^{n}\right\|_{\ell_{2}}$. Therefore, setting $B(n):=\bigcup_{j \in J_{1}(n)} \Delta_{n}^{j}$, we obtain

$$
\begin{aligned}
\left\|\chi_{B(n)} \sum_{i=1}^{n} b_{i} r_{i}\right\|_{1} & =\left\|\sum_{j \in J_{1}(n)}\left(\sum_{i=1}^{n} b_{i} r_{i}\right) \chi_{\Delta_{n}^{j}}\right\|_{1}=\left\|\sum_{j \in J_{1}(n)} \sum_{i=1}^{n} \varepsilon_{i j}^{n} b_{i} \cdot \chi_{\Delta_{n}^{j}}\right\|_{1} \\
& =n^{1 / 2}\left\|\sum_{j \in J_{1}(n)} c_{j} \chi_{\Delta_{n}^{j}}\right\|_{1}=n^{1 / 2} 2^{-n} \sum_{j \in J_{1}(n)}\left|c_{j}\right| \leq n 2^{-n}\left\|\left(b_{i}\right)_{i=1}^{n}\right\|_{\ell_{2}} .
\end{aligned}
$$

Combining this inequality with (16), (17) for $A=B(n)$ and (5), by definition of the norm in the space $\mathcal{M}\left(L_{1}\right)$, we have

$$
\left\|\chi_{B(n)}\right\|_{\mathcal{M}\left(L_{1}\right)} \leq 2 \sqrt{2} n 2^{-n}
$$

Let $\left\{n_{k}\right\}_{k=1}^{\infty}$ be an increasing sequence of positive integers, $n_{k}=2^{m_{k}}, m_{k} \in \mathbb{N}$, satisfying the condition

$$
n_{k}^{1 / 8} \geq 2^{n_{1}+\cdots+n_{k-1}}, \quad k=2,3, \ldots
$$

At first, we construct a sequence of sets $\left\{B_{k}\right\}$. Setting $J_{1}^{1}:=J_{1}\left(n_{1}\right)$ and $B_{1}:=B\left(n_{1}\right)$, in view of (18) we have

$$
\left\|\chi_{B_{1}}\right\|_{\mathcal{M}\left(L_{1}\right)} \leq 2 \sqrt{2} n_{1} 2^{-n_{1}}
$$

To define $B_{2}$, we take for $I_{1}$ any interval $\Delta_{n_{1}}^{j}$ such that $j \notin J_{1}^{1}$. Now, we can choose a set $J_{1}^{2} \subset\left\{1,2, \ldots, 2^{n_{1}+n_{2}}\right\}$ satisfying the conditions: card $J_{1}^{2}=n_{2}, \Delta_{n_{1}+n_{2}}^{j} \subset I_{1}$ for every $j \in J_{1}^{2}$ and the $n_{2} \times n_{2}$ matrix $n_{2}^{-1 / 2} \cdot\left(\varepsilon_{i j}^{n_{1}+n_{2}}\right)_{n_{1}<i \leq n_{1}+n_{2}, j \in J_{1}^{2}}$ is orthogonal. We set $B_{2}:=\bigcup_{j \in J_{1}^{2}} \Delta_{n_{1}+n_{2}}^{j}$. Clearly, $m\left(B_{2}\right)=n_{2} 2^{-\left(n_{1}+n_{2}\right)}$ and $B_{1} \cap B_{2}=\emptyset$, because of $B_{2} \subset I_{1}$. As in the case of $B(n)$ we have

$$
\begin{aligned}
\left\|\chi_{B_{2}} \sum_{i=1}^{n_{1}+n_{2}} b_{i} r_{i}\right\|_{1} & =\left\|\sum_{j \in J_{1}^{2}}\left(\sum_{i=1}^{n_{1}+n_{2}} b_{i} r_{i}\right) \chi_{\Delta_{n_{1}+n_{2}}^{j}}\right\|_{1} \leq\left\|\sum_{j \in J_{1}^{2}}\left(\sum_{i=1}^{n_{1}} b_{i} r_{i}\right) \chi_{\Delta_{n_{1}+n_{2}}^{j}}\right\|_{1} \\
& +\left\|\sum_{j \in J_{1}^{2}}\left(\sum_{i=n_{1}+1}^{n_{2}} b_{i} r_{i}\right) \chi_{\Delta_{n_{1}+n_{2}}^{j}}\right\|_{1} \leq \sum_{i=1}^{n_{1}}\left|b_{i}\right|\left\|\chi_{B_{2}}\right\|_{1}+\left\|\sum_{j \in J_{1}^{2}} \sum_{i=n_{1}+1}^{n_{1}+n_{2}} \varepsilon_{i j}^{n_{1}+n_{2}} b_{i} \cdot \chi_{\Delta_{n_{1}+n_{2}}^{j}}\right\|_{1} \\
& \leq\left(n_{1}^{1 / 2}+1\right) n_{2} 2^{-\left(n_{1}+n_{2}\right)}\left\|\left(b_{i}\right)_{i=1}^{n_{1}+n_{2}}\right\|_{\ell_{2}} \leq n_{2} 2^{-n_{2}}\left\|\left(b_{i}\right)_{i=1}^{n_{1}+n_{2}}\right\|_{\ell_{2}} .
\end{aligned}
$$


Therefore, from (16), (17) and (15) it follows that

$$
\left\|\chi_{B_{2}}\right\|_{\mathcal{M}\left(L_{1}\right)} \leq \sqrt{2}\left(\left(n_{1}+n_{2}\right) 2^{-\left(n_{1}+n_{2}\right)}+n_{2} 2^{-n_{2}}\right) \leq 2 \sqrt{2} n_{2} 2^{-n_{2}} .
$$

Proceeding in the same way, we get a sequence $\left\{B_{k}\right\}$ of pairwise disjoint subsets of $[0,1]$ such that $m\left(B_{k}\right)=n_{k} 2^{-\left(n_{1}+\cdots+n_{k}\right)}$ and

$$
\left\|\chi_{B_{k}}\right\|_{\mathcal{M}\left(L_{1}\right)} \leq 2 \sqrt{2} n_{k} 2^{-n_{k}}, \quad k=1,2, \ldots
$$

Now, define the sets $D_{k}, k=1,2, \ldots$ Select a set $J_{2}^{1} \subset\left\{1,2, \ldots, 2^{n_{1}}\right\}$, card $J_{2}^{1}=n_{1}$, such that each column of the $n_{1} \times n_{1}$ matrix $\left(\varepsilon_{i j}^{n_{1}}\right)_{1 \leq i \leq n_{1}, j \in J_{2}^{1}}$ has exactly one entry equal to -1 and the rest are equal to 1 . Setting $D_{1}:=\bigcup_{j \in J_{2}^{1}} \Delta_{n_{1}}^{j}$, we have $m\left(D_{1}\right)=n_{1} 2^{-n_{1}}$. Furthermore, from the inequality $\left\|n_{1}^{-1 / 2} \sum_{i=1}^{n_{1}} r_{i}\right\|_{1} \leq 1$ (see (5) ) and the definition of $D_{1}$ it follows that

$$
\begin{aligned}
\left\|\chi_{D_{1}}\right\|_{\mathcal{M}\left(L_{1}\right)} & \geq\left\|\sum_{j \in J_{2}^{1}}\left(n_{1}^{-1 / 2} \sum_{i=1}^{n_{1}} r_{i}\right) \chi_{\Delta_{n_{1}}^{j}}\right\|_{1} \\
& =\left\|\sum_{j \in J_{2}^{1}}\left(n_{1}^{-1 / 2} \sum_{i=1}^{n_{1}} \varepsilon_{i j}^{n_{1}}\right) \chi_{\Delta_{n_{1}}^{j}}\right\|_{1} \\
& =\left(n_{1}^{1 / 2}-2 n_{1}^{-1 / 2}\right) n_{1} 2^{-n_{1}} \geq \frac{1}{2} n_{1}^{3 / 2} 2^{-n_{1}}
\end{aligned}
$$

if $n_{1}$ is large enough.

Similarly, we can define the set $D_{2}$. Let $I_{2}$ be any interval $\Delta_{n_{1}}^{j}$ with $j \notin J_{2}^{1}$. Choose the set $J_{2}^{2} \subset\left\{1,2, \ldots, 2^{n_{1}+n_{2}}\right\}$ such that card $J_{2}^{2}=n_{2}, \Delta_{n_{1}+n_{2}}^{j} \subset I_{2}$ for every $j \in J_{2}^{2}$ and each column of the $n_{2} \times n_{2}$ matrix $\left(\varepsilon_{i j}^{n_{1}+n_{2}}\right)_{n_{1}<i \leq n_{1}+n_{2}, j \in J_{2}^{2}}$ has exactly one entry equal to -1 and the rest are equal to 1 . Then, if $D_{2}:=\bigcup_{j \in J_{2}^{2}} \Delta_{n_{1}+n_{2}}^{j}$, then $m\left(D_{2}\right)=n_{2} 2^{-\left(n_{1}+n_{2}\right)}$ and $D_{1} \cap D_{2}=\emptyset$. Moreover, we have

$$
\begin{aligned}
\left\|\chi_{D_{2}}\right\|_{\mathcal{M}\left(L_{1}\right)} & \geq\left\|\sum_{j \in J_{2}^{2}}\left(n_{2}^{-1 / 2} \sum_{i=n_{1}+1}^{n_{1}+n_{2}} r_{i}\right) \chi_{\Delta_{n_{1}+n_{2}}^{j}}\right\|_{1} \\
& =\left\|\sum_{j \in J_{2}^{2}}\left(n_{2}^{-1 / 2} \sum_{i=n_{1}+1}^{n_{1}+n_{2}} \varepsilon_{i j}^{n_{1}+n_{2}}\right) \chi_{\Delta_{n_{1}+n_{2}}^{j}}\right\|_{1} \\
& =\left(n_{2}^{1 / 2}-2 n_{2}^{-1 / 2}\right) n_{2} 2^{-\left(n_{1}+n_{2}\right)} \geq \frac{1}{2} n_{2}^{3 / 2} 2^{-\left(n_{1}+n_{2}\right)} .
\end{aligned}
$$

Arguing in the same way, we construct a sequence $\left\{D_{k}\right\}$ of pairwise disjoint subsets of $[0,1]$ such that $m\left(D_{k}\right)=n_{k} 2^{-\left(n_{1}+\cdots+n_{k}\right)}$ and

$$
\left\|\chi_{D_{k}}\right\|_{\mathcal{M}\left(L_{1}\right)} \geq \frac{1}{2} n_{k}^{3 / 2} 2^{-\left(n_{1}+\cdots+n_{k}\right)}, k=1,2, \ldots
$$

Since $m\left(B_{k}\right)=m\left(D_{k}\right), k=1,2, \ldots$, the functions $f$ and $g$ defined by (15) are equimeasurable ones for arbitrary $\alpha_{k} \in \mathbb{R}, k=1,2, \ldots$ Setting $\alpha_{k}=2^{n_{k}} n_{k}^{-5 / 4}$, by (20), we obtain

$$
\|f\|_{\mathcal{M}\left(L_{1}\right)} \leq \sum_{k=1}^{\infty} \alpha_{k}\left\|\chi_{B_{k}}\right\|_{\mathcal{M}\left(L_{1}\right)} \leq 2 \sqrt{2} \sum_{k=1}^{\infty} n_{k}^{-1 / 4}<\infty
$$


because of $n_{k}=2^{m_{k}}, m_{1}<m_{2}<\ldots$ Thus, $f \in \mathcal{M}\left(L_{1}\right)$.

On the other hand, since $\mathcal{M}\left(L_{1}\right)$ is a Banach function lattice, for every $k=1,2, \ldots$ from (21) and (19) it follows that

$$
\|g\|_{\mathcal{M}\left(L_{1}\right)} \geq \alpha_{k}\left\|\chi_{D_{k}}\right\|_{\mathcal{M}\left(L_{1}\right)} \geq \frac{1}{2} n_{k}^{1 / 4} 2^{-\left(n_{1}+\cdots+n_{k-1}\right)} \geq \frac{1}{2} n_{k}^{1 / 8} .
$$

Hence, $g \notin \mathcal{M}\left(L_{1}\right)$.

\section{References}

[1] S. V. Astashkin, Systems of random variables equivalent in distribution to the Rademacher system and $\mathcal{K}$-closed representability of Banach pairs, Matem. sb. 191(2000). no. 6, 3-30 (Russian); English transl. in Sb. Math. 191(2000), 779-807.

[2] S. V. Astashkin, Rademacher functions in symmetric spaces, Sovrem. Mat. Fundam. Napravl., 32(2009), 3-161 (Russian); English transl. in J. Math. Sci. (N.Y.) (6), 169(2010), 725-886.

[3] S. V. Astashkin and G. P. Curbera, Symmetric kernel of Rademacher multiplicator spaces, J. Funct. Anal. 226(2005), 173-192.

[4] S. V. Astashkin and G. P. Curbera, Rademacher multiplicator spaces equal to $L^{\infty}$, Proc. Amer. Math. Soc. 136(2008), 3493-3501.

[5] S. V. Astashkin and G. P. Curbera, Rearrangement invariance of Rademacher multiplicator spaces, J. Funct. Anal. 256(2009), 4071-4094.

[6] S. V. Astashkin and G. P. Curbera, A weighted Khintchine inequality, Revista Mat. Iberoam. 30(2014), no. 1, 237-246.

[7] C. Bennett and R. Sharpley, Interpolation of Operators, Pure and Applied Mathematics, Vol. 119, Academic Press, Boston, 1988.

[8] G. P. Curbera, Operators into $L^{1}$ of a vector measure and applications to Banach lattices, Math. Ann. 293(1992), 317-330.

[9] G. P. Curbera, A note on function spaces generated by Rademacher series, Proc. Edinburgh. Math. Soc. 40(1997), 119-126.

[10] G. P. Curbera, How summable are Rademacher series?, Operator Theory: Adv. and Appl. 201(2009), 135-148.

[11] J. Diestel, H. Jarchow and A. Tonge, Absolutely Summing Operators, Cambridge University Press, Cambridge, 1995.

[12] W. B. Johnson, B. Maurey, G. Schechtman and L. Tzafriri, Symmetric structures in Banach spaces, Mem. Amer. Math. Soc. No. 217, 1979.

[13] A. Khiintchine, Über dyadische Bruche, Math. Zeit. 18(1923), 109-116.

[14] S. G. Krein, Ju. I. Petunin and E. M. Semenov, Interpolation of Linear Operators, AMS Translations of Math. Monog., 54, Providence, 1982. 
[15] J. Lindenstrauss and L. Tzafriri, Classical Banach Spaces II, vol. 97, Springer-Verlag, Berlin, 1979.

[16] G. G. Lorentz, Relations between function spaces, Proc. Amer. Math. Soc. 12(1961), 127-132.

[17] V. D. Milman and G. Schechtman, Asymptotic theory of finite dimensional normed spaces, Lecture Notes in Mathematics, vol. 1200, Springer-Verlag, Berlin, 1986.

[18] R. E. A. C. Paley and A. Zygmund, On some series of functions. I, II, Proc. Camb. Phil. Soc. 26(1930), 337-357, 458-474.

[19] G. Pisier, Factorization of linear operators and geometry of Banach spaces, Amer. Math. Soc., Providence, RI, CBMS 60, 1986.

[20] V. A. Rodin and E. M. Semyonov, Rademacher series in symmetric spaces, Anal. Math. 1(1975), no. $3,207-222$.

[21] V. A. Rodin and E. M. Semenov, The complementability of a subspace that is generated by the Rademacher system in a symmetric space, Funktsional. Anal. i Prilozhen. (2), 13(1979), 91-92 (Russian); English transl. in Functional Anal. Appl. 13(1979), no. 2, 150-151.

[22] S. J. Szarek, On the best constants in the Khinchin inequality, Studia Math. 58(1976), no. 2, 197-208.

[23] M. Veraar, On Khintchine inequalities with a weight, Proc. Amer. Math. Soc. 138(2011), 41194121.

[24] A. Zygmund, Trigonometric Series. 2nd ed. Vol. I, Cambridge University Press, New York 1959.

Department of Mathematics and Mechanics

Samara State University

Acad. Pavlov, 1

443011 Samara

Russian Federation

E-mail: astash@samsu.ru 